\title{
Quantitative 3D Data Extraction Using Contiguous Volumes ${ }^{1}$
}

\author{
C.J. Dykstra ${ }^{1}$, R. Harrop ${ }^{2}$, Member, IEEE, A.M. Celler ${ }^{1}$, Member, IEEE, and M.S. Atkins ${ }^{2}$ \\ ${ }^{1}$ Div. of Nuclear Medicine, VH\&HSC, 855 West 12th Ave., Vancouver, Canada, V5Z 1M9 \\ ${ }^{2}$ School of Computing Science, Simon Fraser University, Burnaby, Canada, V5A 1S6
}

\begin{abstract}
A new image analysis method, called contiguous volume analysis, has been developed to automatically extract 3D information from emission images. The method considers volumes of activity and displays data about them in a format which allows quantitative image comparison. This method of numerical analysis enables us to show, for example, whether or not information has been gained, lost or changed through the use of different filters and different attenuation and scatter correction, and reconstruction algorithms. Since the analysis method is consistent with a visual inspection of the data, intuitive insights into the meaning of the data are possible, allowing a better understanding of the effects of the different image processing techniques on the images. The method can be used to find patterns of activity in sets of images, and may be used to quantify noise, allowing an objective determination of which volumes in an image are meaningful.
\end{abstract}

\section{INTRODUCTION}

Most emission image analysis, particularly in clinical work, is done by simple visual inspection of the image slices. A great deal information is gained from visual analysis [1], but only of a non-reproducible, qualitative nature. Since many of the images used for diagnostic work are not quantitative, visual analyses most often suffice. However, quantitative single photon emission computed tomography (SPECT) is beginning to appear in the clinics [2], and in order to make use of the additional information, quantitative analyses are needed. For any quantitative work, some form of data extraction is required. Data extraction can be as simple as reading image values under a cursor position, or as complicated as 3D image registration to an anatomic atlas using shifting, rotating, scaling and warping.

It is argued in [3] that a data extraction technique must meet some criteria to be useful. One criterion is reproducibility. If the technique always extracts the same data, regardless of who does the analysis or when it is done, then the method is reproducible. Any fully automated technique can be expected to be reproducible. Another requirement is sensitivity, which is the ability to differentiate between two states. For example, a method

\footnotetext{
${ }^{1}$ We would like to acknowledge the Natural Sciences and Engineering Council of Canada for their financial support.
}

which is designed to differentiate between disease and no disease, and does so, is sensitive to the disease. Sensitivity can only be determined by application of the method to a particular study. A third requirement is that the method has no bias, that is, no tendency either toward or away from any particular result.

By far the most common data extraction method in SPECT and positron emission tomography (PET) is to use 2- or 3D volumes of interest (VOI) and then extract the data from those volumes (as in $[4,5,6,7]$ ). Using hand-drawn volumes ensures that the investigator gets exactly the data wanted, but it is very slow, has reproducibility and bias problems and it is impractical to attempt for extracting data from an entire $3 \mathrm{D}$ image. Automatic methods of VOI definition, such as cortical circumferential profiling for PET data $[8,9]$, are faster and provide reproducible data, but require good understanding of the method to ensure that the data extracted is indeed applicable to the study at hand. Anatomical interpretations of the data must be made with care.

Anatomical volume definition, through registration to an anatomical atlas, is reproducible, but is dependent on the accuracy of the registration technique [10]. The scarcity of anatomical landmarks in emission data makes registration a difficult problem and it is hard to identify errors which may have been introduced and to determine what effect they will have on the image analysis. Anatomical VOIs are used in functional anatomy research in order to gain the necessary information about underlying anatomy. Such work is usually done with activation studies, where an at-rest image is compared to an activated image using a statistical analysis in order to find volumes of significant difference (for example, $[11,12,13])$. One difficulty with these functional analyses is that they often require image averaging in order to improve the signal-to-noise ratio enough so that differences between the images can be found. While useful for functional anatomy research, such methods are not directly usable in clinical work since each patient must be analyzed individually.

To answer this need for a general, automated data extraction technique which is appropriate for analysis of individual PET or SPECT images, we developed the contiguous volume analysis. Our technique was specifically designed to find and extract the data that physicians use on a daily basis. In most studies it is the areas and volumes of high activity which are of most 
interest, and it is their sizes, values and positions which are wanted. Contiguous volume analysis automatically finds all of the hot volumes in the entire 3D image. The values and sizes of all of the distinct hot spots in the image are saved and then plotted on a graph, allowing immediate appraisal of all the significant hot spots throughout the entire 3D data set. Full automation ensures that the method is reproducible. Since no decisions are made about the image during the extraction process, there is no bias in the extracted data and the information is plotted in a way that clearly shows the significance of any volume, removing much of the bias from the image interpretation as well. Since the method parallels visual inspection, it is appropriate for use with any study where visual inspection would be used, as well as with quantitative studies where visual inspection is useful but not sufficient. The extracted data can be used for automatic VOI definition, quantitation and for interactive image analysis.

Although the idea of contiguous volume analysis was introduced a number of years ago [14], and some of its applications have been presented in abstracts $[15,16]$, a complete discussion of the method is not generally available. We therefore intend, in this paper, to present a full explanation of the method and briefly discuss applications for it which we currently have in mind.

\section{METHOD}

The contiguous volume method can be likened to creating a topographical map of a mountainous landscape. A series of elevations is chosen and at each elevation, the contour of landscape is determined and drawn. At a single elevation, the contour lines show how many mountains are at least that high, the area they cover, and where they are. A complete set of contour lines, over the full range of elevations, provides a great deal of information about the topography of the landscape.

For emission images, the data is usually $3 \mathrm{D}$, not $2 \mathrm{D}$, and therefore 3D volumes are found at each step, not areas, but otherwise the methods are much the same. ${ }^{2}$ The program starts at the highest image value and decreases the threshold by a user-specified step size until the minimum image value is reached. At each threshold (elevation), the image is searched for all distinct hot spots which exist above the threshold (mountain tops). Each connected set of voxels found at one particular threshold is called a volume (single mountain top). The size, range of voxel values and position ( $\mathrm{x}, \mathrm{y}$ and $\mathrm{z}$ coordinates of the centroid) of each volume is kept. As the program steps through decreasing thresholds, volumes found at one threshold are compared to those found at the previous

\footnotetext{
${ }^{2}$ For simplicity, in this paper the image elements will always be referred to as voxels, and the connected regions as volumes, regardless of whether the image is $2-$ or $3 D$. Since the method works in exactly the same way on 2 - and $3 D$ data, the distinction is not important.
}

threshold. Each volume is either a new hot spot which did not exist before, a hot spot which has grown from one found at the previous threshold, or one that has resulted from the merging of two or more hot spots found previously. The information about the hot spots appearing, growing and merging is accumulated into sequences. A sequence is a nested set of volumes found at consecutive thresholds which, together, describe a distinct hot spot in the image. The final volume in each sequence describes the entire hot spot and previous volumes in the sequence provide information about the location and threshold at which the sequence first appeared and how it grew. Just as there are no overlapping lines in a contour map, there are no overlapping volumes or sequences in an image.

The entire image, from the image maximum down to its minimum, is characterized by volumes (set of connected voxels found at one particular threshold) and sequences (nested series of volumes which define one distinct mountain). It is possible for a sequence to consist of only one volume, if it immediately merges with another sequence at the next threshold after it was formed, or for it to persist through many thresholds (like a single mountain standing on a plain). The size of the final volume at the image minimum will be equal to the size of the entire data set.

Once all of the sequences in an image have been found, the data can be plotted in a graph of volume vs. threshold, called a Feature Analysis (FA) graph (see Fig. 1). Each sequence is plotted as a polygon indicating the range of values in the sequence and its size at each threshold. At a threshold where previously distinct volumes have merged into a new, single volume, the previous sequences are plotted side by side, largest leftmost, on top of the new sequence that they form. A simple example of how the FA graph is formed is given in Fig. 1, and Fig. 2 shows the method for a 2D slice taken from an 3D SPECT image.

The FA graph does not plot a true function, where each $x$ value corresponds to exactly one $y$ value, therefore it cannot be read like a normal graph. Values can be read directly off the vertical axis, but the horizontal axis is used like a ruler. For example, the volume of sequence II in Fig. 1 is found by finding the range over which it is plotted, i.e. from 238 to 321 , indicating that the connected volume contained 83 voxels at the 100 threshold.

Since the FA graph cannot show spatial information, some means of interacting with the slice image is necessary in order to match the spikes in the graph with the hot spots in the image. To answer this need, a graphical user interface has been written which allows the user to interact with the slice display and FA graph. The interface allows the user to select sequences in the FA graph and see the corresponding hot spot highlighted in the image, or to select a point in the image and see both the image volume and the sequence in the FA graph highlighted. When either of these operations is performed, information about 

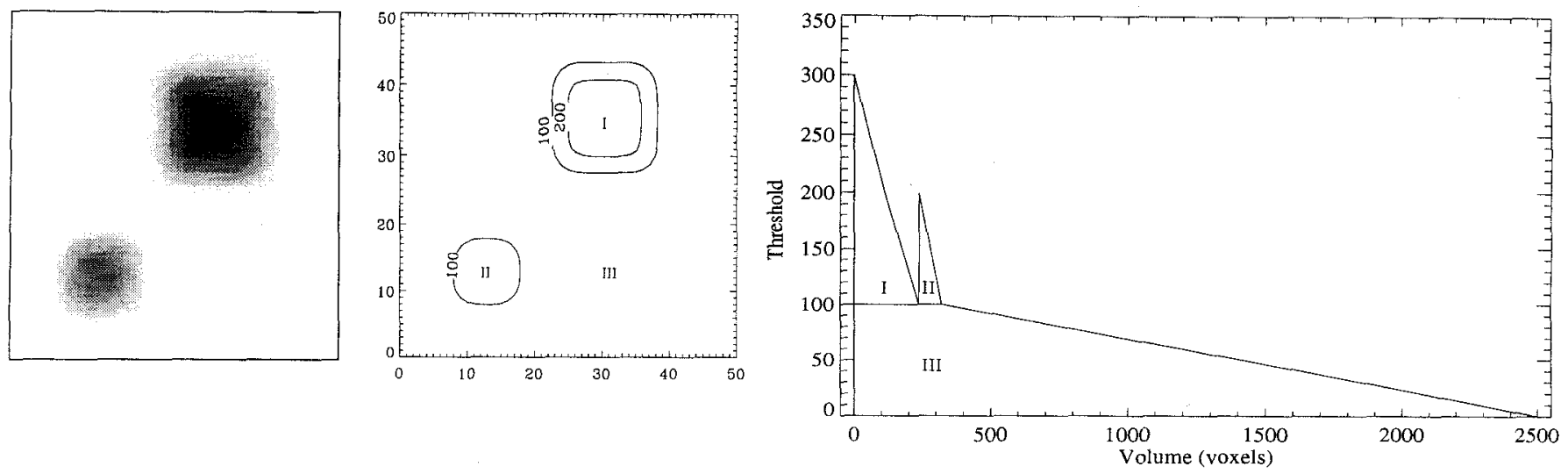

Fig. 1 Simple 2D example of the contiguous volume algorithm. On the left is the image with two hot sources. In the middle is the contour map of the image showing the two thresholds at which the contiguous volumes were found for this example. The volumes found at these two thresholds were used plot the feature analysis graph, shown on the right. At the top threshold, 200, only the hottest volume, I, is found; it contains 112 voxels and has a maximum value of 300. At the next threshold, 100 , two hot spots are found, I, which has grown to 238 voxels, and a new hot spot, II, which has 83 voxels and a maximum of 199 . At the last threshold, 0, the entire 50x50 image is found, making a single volume of 2500 voxels, labeled III. Each of. the labeled volumes in the slice image corresponds to a sequence, I and II are hot spots and III is created by their merging at the last threshold. This sequence data is plotted in the FA graph, on the right. Sequence I is plotted as the left-most spike. The tip of the spike shows the hottest voxel in the sequence, 300, the base of the peak shows the last threshold at which it existed as a distinct hot spot, 100, and its sizes at the two thresholds at which it was found, 112 and 238 , are shown by the width of the spike at those thresholds. Since sequence II exists at some of the same thresholds as sequence I did, it must be plotted beside the other. Sequence I is plotted leftmost simply because it has the most voxels. Sequence II starts at 238 on the $x$-axis, and goes to $238+83=321$, the $\mathrm{x}$-axis is thus used as a ruler to measure the sizes of the sequences in the image. The spike labeled II in the graph shows that the hottest value in that sequence was 199 , that it had 83 voxels at a threshold of 100 and that it merged with sequence I to form sequence III, which has 2500 voxels, by the final threshold, 0 .

the sequence selected, such as size and range of values, is displayed.

\section{DISCUSSION}

Although the contiguous volume algorithm and the FA graph can be somewhat difficult to understand, the extracted data is not. The meaning of the hot spots is straightforward, and people who are familiar with emission images typically have no difficulty understanding the extracted data and generally have immediate suggestions for its use. The graphical interface also aids understanding by demonstrating the direct correspondence between the sequences and the image. Since the method parallels visual inspection, intuitive insights into the meaning of the data are possible.

The main advantage of having the FA graph available along with the slice image is that it clearly provides information which is difficult to extract from the slice image, namely image values. Although the human visual system is extremely good at recognizing patterns and shapes from limited data, it cannot distinguish between many grey- or colourscale values. It is these values which are readily available from the FA graph; they are provided in the context of hot spots and volumes of interest which are of most use to the observer. The FA graph also provides information about 3D volumes, which can be difficult to get from a visual inspection of slices. The exact distinction between any hot volume and its background is also easily seen, something which, in the slice display, is dependent on the colourscale. This information is also available from profiles, but only in one dimension. With profiles, the users must decide which are the most useful lines to plot since it is inconvenient to plot many, and must be confident that nothing of interest has been missed. The FA graph shows the data from the full 3D image so that there cannot be anything missed.

We have observed that the FA graph is useful in avoiding bias in the interpretation of images. For example, the slice images of the Jaszczak phantom shown in Figs. 3 and 4 show five hot spots corresponding to the five largest spheres; the FA graphs, however, clearly show only the four largest, the fifth is not apparent. The reason for this is that there is noise in the rest of the image which is similar in size and value to the fifth sphere. Since we see a hot spot where we expect the fifth sphere to be, we assume that it has been recovered by the reconstruction and ignore the other hot spots of similar size and intensity since we know they must be noise. However, the FA graphs clearly show that some noise is of equal size to the fifth sphere and that it therefore cannot be considered to have been completely recovered. If the location of the fifth sphere were not known in advance, it would not be possible to positively identify it, for example, if the four largest spheres are covered, the fifth becomes indistinguishable from the background. 

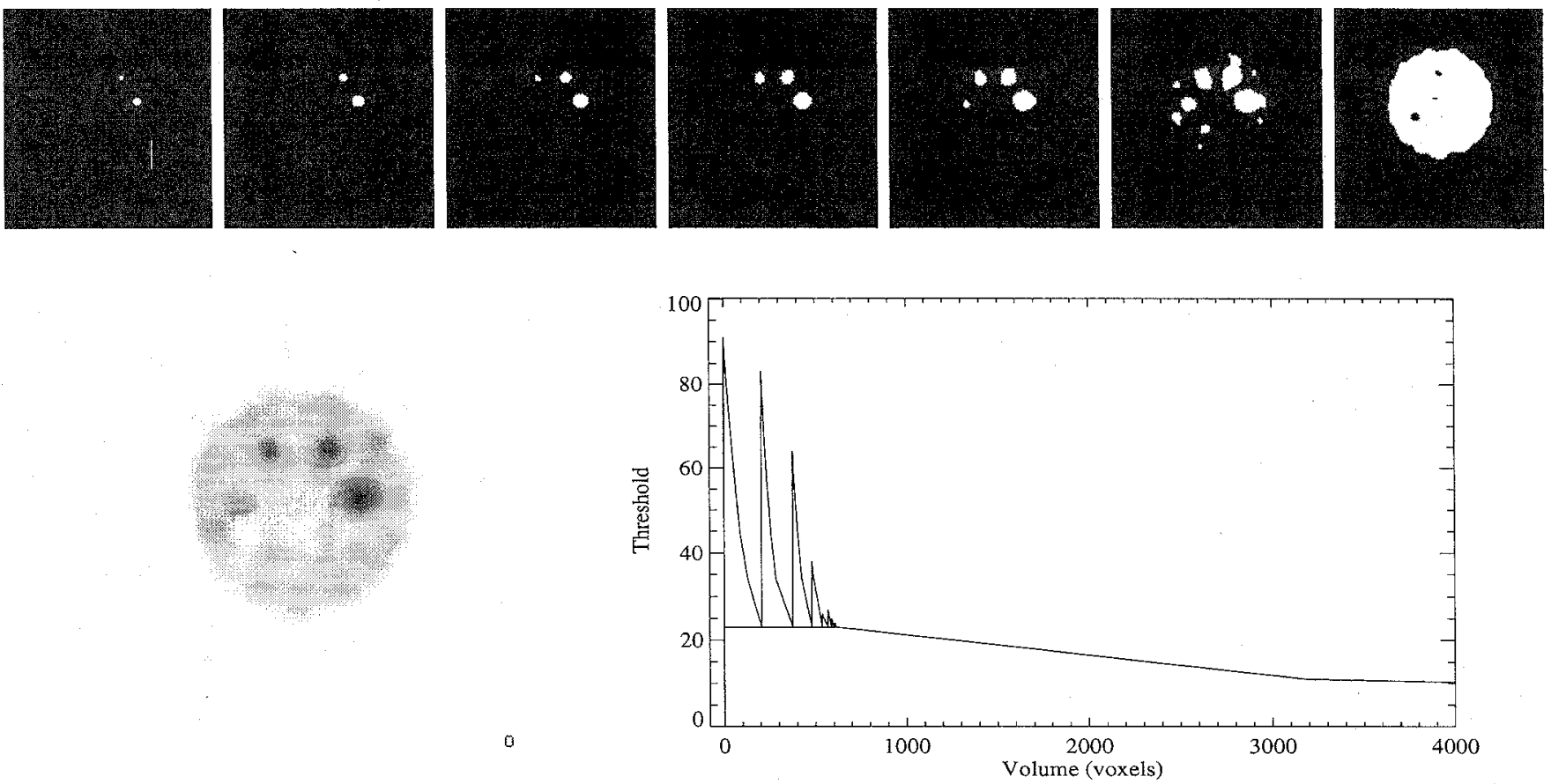

Fig. 2 A 2D example of thresholding and the FA graph for a single horizontal slice taken from a 3D reconstruction of a Jaszczak phantom. The FA graph was generated using just eight thresholds on the image. The top row of binary images show the slice at the first seven thresholds, clearly showing the contiguous volumes found at each (the eighth is 0 where the entire image is found). The slice image (numbered 0) is shown bottom left, and the FA graph bottom right. The FA graph has been cut off at 4000 voxels in order to be able to see the detail of the sequences which are concentrated at the left. Beyond 4000 voxels, the graph just smoothly extends to 16384 voxels, the dimensions of the $128 \times 128$ slice.

Such information about the size of noise throughout the image volume is useful in determining how much significance should be attached to any individual hot spot. Also, since the meaning of the extracted volumes is straightforward, it can be easily factored in with other diagnostic information for clinical decision making.

The FA graph can be used for simple, interactive VOI definition and analysis. Selecting a VOI is simply a matter of displaying the image and the FA graph, and using the interface to find and highlight various hot spots. The information about the size, value and position of the VOI is displayed and the volume is marked in the slices. The user can easily check all the significant regions in the image by choosing the distinct peaks in the FA graph and seeing which regions are highlighted in the image.

The method could be quite useful for the quantitative analysis of images. An example is given here of its application to a study on the effect of different digital filters on 3D SPECT images [16]. Figs. 3 and 4 are two reconstructions of a Jaszczak phantom with six hot spheres in a uniform, warm cylinder; the ratio of activity in the spheres to the background was 7.7. Fig. 3 was reconstructed with a Hanning filter (cutoff 0.5) and Fig. 4 with a Butterworth filter (cutoff 0.2 , order 5). The Hanning filter is a strongly smoothing filter which removes much of the noise and provides a smooth image but, as is shown by the FA graph, the voxel values in the spheres are low (sphere to background ratio of the two largest is about 5.3) and the reconstructed volume is enlarged (recovered volumes are 3 to 4 times actual size). A much noisier image is produced by the Butterworth filter, but it does a better job of recovering the values in the spheres (sphere to background ratio of the two largest is about 6.3) and volumes of the spheres (recovered volumes are 1 to 2 times actual size). In both of these images, although the fifth sphere can be seen in the images, the FA graph shows that there is noise in the image of equivalent size and value, indicating that neither filter was able to completely recover the fifth sphere. Using profiles to try to gain the same information is not possible unless the highest noise happens to lie on the line which was chosen to measure the fifth sphere.

The ease with which it is possible to determine the volumes and values in hot spots, and to test if small regions have been fully recovered makes this analysis useful for the comparison of different image correction and reconstruction techniques algorithms. The Medical Imaging Research Group at Vancouver Hospital \& Health Sciences Centre is currently developing a unified method for quantitative SPECT $[17,18]$. The use of a precise, quantitative analysis is essential for determination of the effects of different image processing techniques on the final image, as well as for clinical analysis of the final images. 

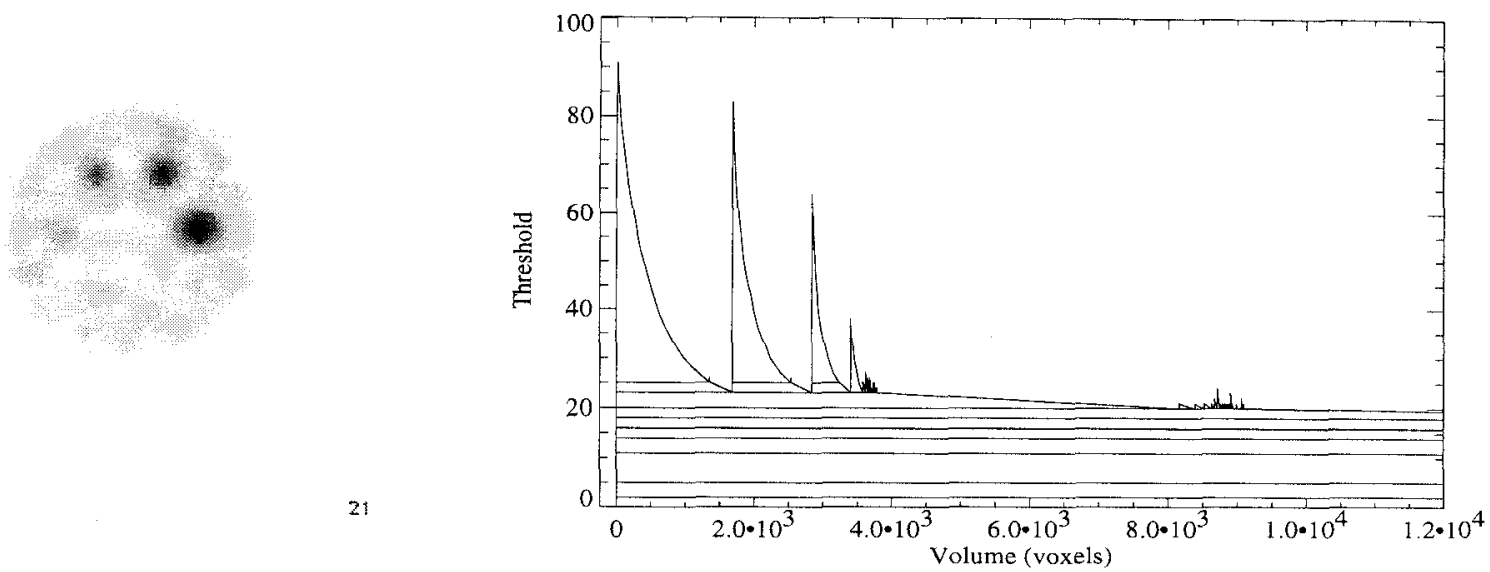

Fig. 3 Jaszczak phantom of 6 hot spheres in a warm background, imaged on a Sopha DST SPECT camera and reconstructed with a Hanning filter (cutoff 0.5). Slice 21 from the $128 \times 128 \times 64$ image is shown here with the FA graph for the full 3D data set. The sequences for the graph were found using 40 thresholds. The FA graph has been cutoff at 12,000 voxels on the $\mathrm{x}$-axis since the data of interest is concentrated in this region; the full graph extends to 1,048,576 voxels, the size of the entire image. Beyond 12,000 voxels, the main volume continues to merge with noise as the threshold is dropped, but there are no more significant volumes.
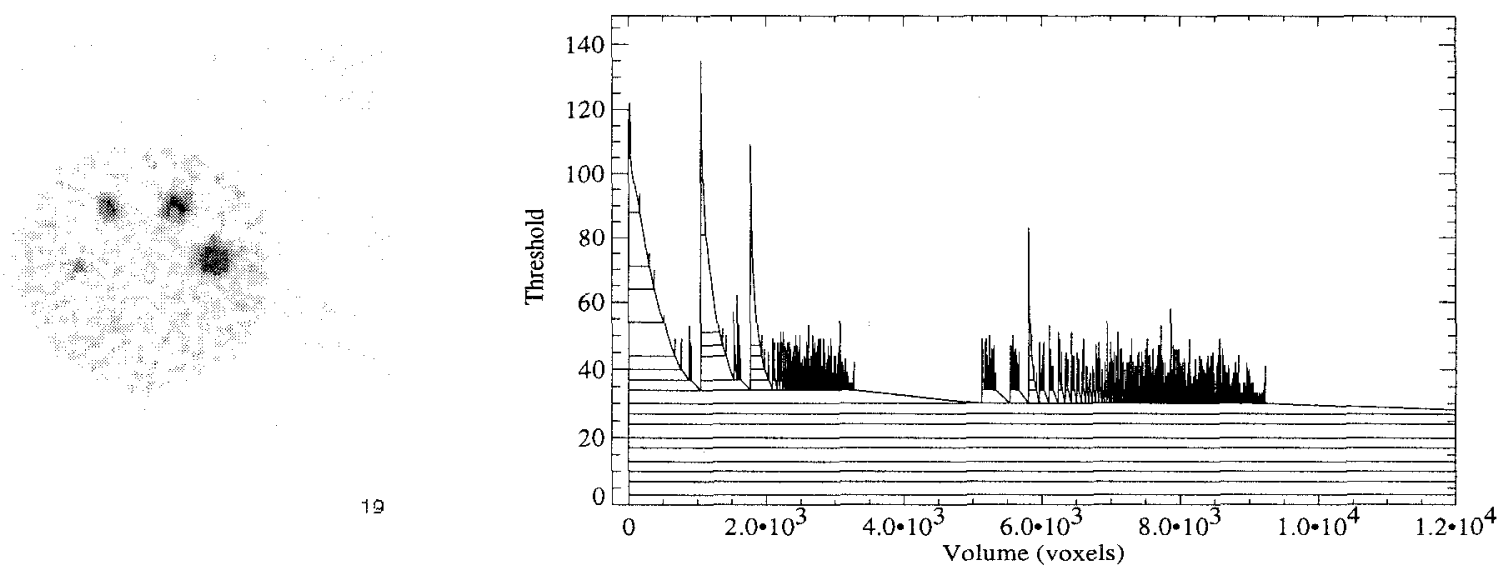

Fig. 4 Jaszczak phantom reconstructed with a Butterworth filter (cutoff 0.2, order 5). Slice 19 from the $128 \times 128 \times 57$ image is shown with the the FA graph for the full $3 \mathrm{D}$ data set. The sequences for the graph were found using 40 thresholds. The FA graph has been cutoff at 12,000 voxels on the $\mathrm{x}$-axis since all of the data of interest is concentrated in this region; the full graph extends to 933,888 voxels.

Some data sets are characterized by a consistent arrangement of hot spots, PET FDG brain studies for example. In normal images, certain hot spots are always visible, such as the visual cortex, thalami and basal ganglia. A pattern of such hot spots can be defined as a set of sequences which consistently appear in a particular spatial arrangement [15]. Disease is shown by the differences between the normal pattern of hot spots and the arrangement found in a disease study.

A particularly interesting use of the method may prove to be in the analysis of noise. Since all the small hot spots have been identified, they can be removed from the image. However, doing this has scarcely any effect on the image, as can be seen from a comparison of Figs. 4 and 5. The removal of these regions from the FA graph has a much greater effect since they are more clearly visible in this type of display. Removal of all small hot spots from the graph means that the analysis of the image will automatically ignore such noise.

For the phantom examples used in this paper, the definition of noise is simple; anything which is not one of the six hot spheres. In this data, the sizes of the two smallest spheres overlap with noise. When analyzing patient data, however, the distinction is not so clear since the correct distribution of activity is not known. Given the camera parameters, automatic determination and removal of all hot spots smaller than the camera resolution is simple. Automatic determination of the rest of the statistical noise is more difficult; work has been done on the evaluation of local statistics within an VOI [19] which may be usable with our method and a method of automatically determining the level of noise in images 


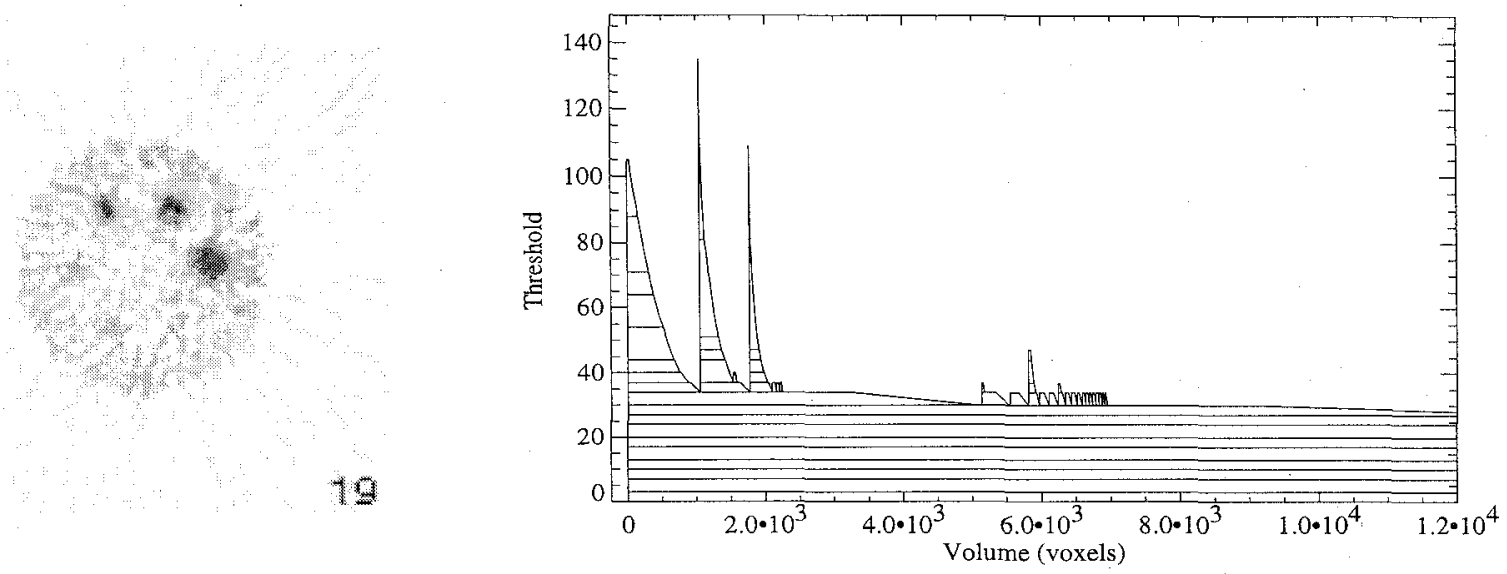

Fig. 5 Example of noise removal on the Jaszczak phantom image reconstructed with the Butterworth filter. In this $3 \mathrm{D}$ image, all hot spots with volumes less than or equal to 33 voxels were removed (ie. all hot spots less than the machine's best possible resolution). The effect on the slice image is hardly noticeable since these small hot spots are not very apparent to the human eye in this type of display. The effect on the FA graph, however, is immediately apparent (14303 out of 14639 sequences were removed), making the significant spikes in the graph much easier to read.

[20] may also prove useful.

When analyzing images with low counts, it is often difficult to distinguish between noise and meaningful regions of elevated activity. The FA graph can be used to identify these regions even though they do not form a coherent, distinct peak. For example, the uniform, warm cylinder of the Jaszczak phantom shown in Figs. 3, 4 and 5 always appears as a mottled, extremely irregular volume until the threshold drops below the lower values in the cylinder. The irregular volume indicates a general area of elevated activity in the image. In extremely low count data sets, individual hot spots may never reach a meaningful size, but such general areas of elevated activity are nevertheless identifiable. Clearly a sequence must reach a much larger size before it can be argued that it shows the existence of a region of higher counts, but it need not form a distinct peak. Such regions are simple to find once the noise has been removed from the FA graph.

Our method of data extraction is general and should have wider application beyond emission image analysis. It is appropriate for use with data where regions of high (or low) activity are of interest. It has, for example, been applied to radiotherapy planning, to check for cold spots in the tumor or hot spots in the organs-at-risk. The likelihood of success, and of complication, of a treatment is strongly dependent on the sizes and positions of, and dose levels in, hot or cold spots present in the dose plan. Contiguous volumes have been used to determine and conveniently display this information [21].

\section{CONClusions}

The contiguous volume method is based on visual analysis, and is designed to automatically extract the same elements from an image as a visual inspection. The advantages of this approach are that 1) the method is, in many cases, as broadly applicable as visual inspection, with the ability to extract quantitative informations and reproducibility of an automated technique, 2) the meaning of the extracted data is straightforward and therefore can be easily factored in with other diagnostic information during clinical decision making, and 3) no decisions are made about the image during the data extraction process, so the method is unbiased. The FA graph represents the hot spot information in such a way that the significance of each region is clearer than in the image slice display, which removes much of the bias from image interpretation as well. We believe this method of data extraction is useful and has considerable potential in the field of emission image analysis.

\section{REFERENCES}

[1] G. D. Chiro and R. Brooks, "PET quantitation: blessing and curse.," J. Nuc. Med., vol. 29, no. 9, pp. 1603-1604, 1988. Editorial

[2] M. Rosenthal, J. Cullom, W. Hawkins, S. Moore, B. Tsui, and M. Yester, "Quantitative SPECT imaging: A review and recommendations by the focus committee of the Society of Nuclear Medicine Computer and Instrumentation Council," J. Nuc. Med., vol. 36, pp. 1489 1513, 1995.

[3] C. Clark, "Minority opinion on the extraction of regional values from functional brain images," J. Cerebral Blood Flow and Metabolism, vol. 7, no. 12, pp. S13-S16, 1987.

[4] T. Adair, P. Karp, A. Stein, R. Bajcsy, and M. Reivich, "Computer assisted analysis of tomographic images of the brain," J. Computer Assisted Tomography, vol. 5, no. 6, pp. 929-932, 1981. Technical note.

[5] R. Bajcsy, R. Leiberson, and M. Reivich, "A computerized system for the elastic matching of deformed radiographic images to idealized atlas images," J. Computer Assisted Tomography, vol. 7, no. 4, pp. 618-625, 1983.

[6] A. Evans, S. Marrett, L. Collins, and T. Peters, 
"Anatomical-functional correlative analysis of the human brain using three dimensional imaging systems," in SPIE Medical Imaging III: Image Processing, vol. 1092, pp. 264274, 1989.

[7] M. Posner, S. Petersen, P. Fox, and M. Raichle, "Localization of cognitive operations in the human brain," Science, vol. 240, pp. 1627-1631, 1988.

[8] E. D. Bella, G. Gullberg, A. Barclay, and R. Eisner, "Circumferential profiling for region-based analysis of dynamic SPECT data," in 1996 IEEE Nuclear Science Symposium and Medical Imaging Conference Record, 1996. To appear.

[9] J. Links, H. Loats, H. Holcomb, S. Loats, M. Stumpf, and H. W. Jr., "Cortical circumferential profiling: an objective approach to cortical quantification in emission tomography," in Proceedings of the 36th Annual Meeting, vol. 30, (St. Louis, Missouri), p. 816, Society of Nuclear Medicine, 1989.

[10] W. Drevets, T. Videen, A. MacLeod, J. Haller, and M. Raichle, "PET images of blood flow changes during anxiety: correction," Science, vol. 256, p. 1696, 1992.

[11] K. Friston, C. Frith, P. Liddle, R. Dolan, A. Lammertsma, and R. Frackowiak, "The relationship between global and local changes in PET scans," J. Cerebral Blood Flow and Metabolism, vol. 10, no. 4, pp. 458-466, 1990.

[12] C. Clark and R. Carson, "Analysis of covariance in statistical parametric mapping," J. Cerebral Blood Flow and Metabolism, vol. 13, no. 6, pp. 1038-1040, 1993. Letter to the Editor.

[13] K. Worsley, A. Evans, S. Marrett, and P. Neelin, "A threedimensional statistical analysis for $\mathrm{CBF}$ activation studies in human brain," J. Cerebral Blood Flow and Metabolism, vol. 12, no. 6, pp. 900-918, 1992.

[14] C. Dykstra, R. Harrop, and M. Atkins, "3D analysis for positron emission tomography of the brain," in Proceedings of the International Conference on Volume Image Processing, (Utrecht), pp. 103-106, 1993.

[15] C. Dykstra, R. Harrop, and M. Atkins, "Defining a 3D pattern for normal PET images," Clinical Nuclear Medicine, vol. 18, no. 10, p. 931, 1993. Abstract.

[16] C. Dykstra, R. Harrop, and A. Celler, "Quantitative data analysis for emission imaging," Med. Phys., vol. 23, p. 810, May 1996. Abstract.

[17] A. Celler, A. Sitek, and R. Harrop, "Reconstruction of multiple line source attenuation maps," in 1996 IEEE Nuclear Science Symposium and Medical Imaging Conference Record, November 1996. To appear.

[18] R. Wells, A. Celler, and R. Harrop, "Experimental validation of an analytical method of calculation of photon distributions," in 1996 IEEE Nuclear Science Symposium and Medical Imaging Conference Record, pp. 1402-1406, November 1996.

[19] X. Yu, C. Huang, R. Leahy, J. Bading, and P. Conti, "Analytic computation and sample estimation of the local statistics in positron emission tomography images: a comparative study," J. Nuc. Med. - Supplement, vol. 37, no. 5, p. $155 \mathrm{P}, 1996$.

[20] J. Beis, A. Celler, and J. Barney, "An automated method to determine cutoff frequency based on image power spectrum," IEEE Trans. Nuc. Sci., vol. 42, no. 6, pp. $2250-$ $2254,1995$.

[21] C. Dykstra, T. Bortfeld, J. Debus, A. Lomax, R. Harrop,
W. Schlegel, and G. Munkel, "Characterization of dose distribution in radiation therapy plans," Radiotherapy and Oncology, vol. 41, pp. 281-284, 1996. Technical Note. 